\title{
Factores determinantes de la probabilidad de incumplimiento tributario en América Latina
}

Espinosa, Alexandra M.; Sarmiento, Alex

Factores determinantes de la probabilidad de incumplimiento tributario en América Latina

Revista Economía y Política, núm. 31, 2020

Universidad de Cuenca, Ecuador

Disponible en: http://www.redalyc.org/articulo.oa?id=571162102004

DOI: https://doi.org/10.25097/rep.n31.2020.02

La Universidad de Cuenca en Ecuador, conserva los derechos patrimoniales (copyright) de las obras publicadas, y favorece y permite la reutilización de las mismas bajo la licencia Creative Commons Atribución-NoComercialCompartirlgual 4.0 Internacional (CC BY-NC-SA 4.0), por lo cual se pueden copiar, usar, difundir, transmitir y exponer públicamente, siempre que: a. Se cite la autoría y fuente original de su publicación (revista, editorial, URL y DOI de la obra). b. No se usen para fines comerciales u onerosos. c. Se mencione la existencia y especificaciones de esta licencia de uso.

Esta obra está bajo una Licencia Creative Commons Atribución-NoComercial-Compartirlgual 4.0 Internacional. 
Artículos

\section{Factores determinantes de la probabilidad de incumplimiento tributario en América}

\section{Latina}

Determining factors of the tax non-compliance probability in Latin America

Alexandra M. Espinosa

Escuela Politécnica Nacional, Ecuador

alexandra.miranda@epn.edu.ec

DOI: https://doi.org/10.25097/rep.n31.2020.02

Redalyc: http://www.redalyc.org/articulo.oa?

(DD http://orcid.org/0000-0001-6033-9813

Alex Sarmiento

Escuela Politécnica Nacional, Ecuador

asarmiento@solidario.fin.ec

(iD http://orcid.org/0000-0002-5709-7398

Recepción: 28 Junio 2019

Aprobación: 10 Diciembre 2019

\section{Resumen:}

Este trabajo analiza la relación entre el cumplimiento tributario en América Latina y los determinantes personales e institucionales de la probabilidad de incumplimiento tributario. Así, el principal logro de este estudio es el análisis de los factores socioeconómicos, demográficos e institucionales que modifican la probabilidad de no cumplimiento. La metodología empleada es un modelo de elección binaria Probit con indicadores construidos con ACM. A pesar de que el trabajo no se centra en discutir la confianza de las instituciones, un importante resultado es la formulación de la conjetura sobre el concepto de credibilidad respecto a la confianza en las instituciones en América Latina.

Palabras clave: Moral fiscal, Cumplimiento tributario, Credibilidad, América Latina.

\section{Abstract:}

This article analyses the relationship between tax compliance and the personal and institutional determinants of the probability of non-compliance in Latin America. Therefore, the fundamental achievement of this paper is the analysis of socioeconomic, demographic and institutional factors that modify the probability of non-compliance. The methodology used is a Probit binary choice model with indicator variables constructed using the MCA technique. Although this work is not focused on discussing the reliability of the institutions, an important result obtained is the formulation of conjecture regarding the concept of trustworthiness concerning institutions in Latin America.

KeYwORDs: Fiscal morale, Tax Compliance, Credibility, Latin America.

\section{INTRODUCCIÓN}

La motivación para el cumplimiento tributario ha llevado a numerosas investigaciones que cubren diferentes áreas del conocimiento y disciplinas, tales como la Contabilidad, la Economía, las Ciencias Políticas, la Administración Pública y la Psicología (Saad, 2012). En términos generales, el cumplimiento tributario se refiere a la predisposición (deseo no forzado) de un individuo a actuar de acuerdo con las reglas tributarias (Saad, 2012).

La teoría tradicional sobre el cumplimiento tributario, más conocida como modelos de disuasión, se basa en el trabajo seminal de Allingham y Sandmo (1972), que establece que ninguna persona está dispuesta a pagar impuestos voluntariamente. Estos estudios, fuertemente vinculados a la economía del crimen, niegan la posibilidad de que un individuo desee pagar impuestos de forma voluntaria. De este modo, la única 
política tributaria efectiva es la persecución de la evasión mediante inspecciones y sanciones que disuadan al contribuyente de la comisión del delito o falta administrativa.

Sin embargo, los estudios empíricos muestran que los modelos de disuasión tienen un rendimiento pobre para predecir el cumplimiento tributario (Torgler, 2007). La clave del debate está en la misma definición del concepto de evasión fiscal. El verbo evadir implica que el contribuyente se encuentra "dentro" y, por lo tanto, sólo se puede evadir el pago de un impuesto cuando se tiene la obligación tributaria, es decir, cuando existe el hecho imponible y una deuda tributaria. Así, la evasión, en sentido estricto, supone la omisión o comisión de actos que eluden de forma culposa o negligente el deber legal de tributar.

Sin embargo, por moral tributaria se debe entender el reconocimiento del deber como algo "bueno" o "legítimo", anterior inclusive a la existencia del hecho imponible y la deuda tributaria. Bajo esta definición, un sistema coercitivo o disuasorio puede no ser suficiente para garantizar que los individuos reconozcan el deber de pagar impuestos. Así, bajo una vertiente más moral, más importante que perseguir a los defraudadores en un clima de "policías y ladrones", lo esencial es lograr que los individuos reconozcan la legitimidad del acto y contribuyan voluntariamente, como una obligación ética o moral, más allá de la legalidad vigente.

Por ello, este trabajo va más allá de la evasión fiscal, e incluye indirecta y sutilmente la ingeniería fiscal o el uso de instrumentos legales para reducir la carga tributaria. La ingeniería fiscal no es fraude ni evasión, sino simplemente incumplimiento tributario. Así, el no reconocimiento del deber tributario puede implicar que los contribuyentes traten de pagar menos impuestos de forma legal, donde no existe evasión fiscal propiamente dicha, pero sí incumplimiento del deber de contribuir con los gastos comunes del país.

Este artículo se distribuye de la siguiente manera. En la segunda sección, se analiza brevemente el marco teórico relacionado con la teoría del cumplimiento y los sistemas tributarios en América Latina. En la sección 3, discutimos los datos y la metodología utilizados en este estudio. En la sección 4, se estima la probabilidad de cumplimiento tributario dados los factores determinantes de la moral tributaria con un modelo de elección binaria Probit y se discuten los resultados. Finalmente, en la sección 5, se discuten a profundidad los resultados.

\section{MARCO TEÓRICO}

En el marco de la constitución política romana, se perfilan las ideas de potestas y auctoritas. La autoridad se define como una prerrogativa de ciertos sujetos dotados de carisma y prestigio personal, investidos de una razón superior. La autoridad es un poder superior (asimilable a persuasión), basado en una ética pública reconocida por la comunidad. En cambio, la potestad representaría el poder humano (disuasión), establecido por los hombres para la conducción de la comunidad, y que es definido por el uso de la fuerza o la coacción (Casinos Mora, 1999).

La moral tributaria se adentra en este espinoso terreno del fundamento del derecho, vinculando el cumplimiento tributario con el binomio autoridad-potestad. Así, una autoridad tributaria perfecta tendría por objetivo fundamental ayudar (persuadir) a los contribuyentes a cumplir de forma voluntaria con este deber legítimo. En este mundo ideal, el uso de instrumentos coercitivos (disuasión) sería una excepción dentro de las funciones de la autoridad tributaria perfecta.

Dentro de este binomio disuasión-persuasión o autoridad-potestad, los trabajo alineados con la moral tributaria muestran que la disuasión por sí sola no es suficiente para garantizar el cumplimiento tributario (Saad, 2012), aunque sí necesaria (Braithwaite, 2007). La persuasión, entendida como el conjunto de instrumentos destinados a mejorar la moral tributaria, puede depender directamente de la autoridad tributaria (factores institucionales), o ser inherentes al contribuyente (factores demográficos, socioeconómicos y culturales).

A nivel institucional, Kirchler et al. (2008) establecen dos factores determinantes de moral tributaria: el empoderamiento del Estado entendido como la percepción de la capacidad de la autoridad tributaria para 
detectar y castigar a los defraudadores; y la confianza general de que la autoridad tributaria asume el rol de proveedor de bienes públicos de calidad (clima de servicio), o que actúa en pro del bien común (clima de confianza).

El diseño tributario y el ejercicio del binomio autoridad-potestad por parte de la autoridad tributaria se asumen endógenos al cumplimiento tributario. El uso adecuado del poder coercitivo, con sanciones creíbles, sustentado sobre un poder legítimo son una herramienta efectiva para alcanzar niveles de confianza más altos (Bergman, 2003). Así, el poder coercitivo inapropiadamente utilizado produce un clima antagónico, caracterizado por la desconfianza y el resentimiento, que conduce a un círculo vicioso en el cual el poder coercitivo y la desconfianza se refuerzan mutuamente. Saad (2012) muestra que la complejidad del sistema impositivo es otra razón fundamental para incumplimiento tributario.

Sin embargo, retomando el binomio potestad-autoridad, las buenas prácticas tributarias y un buen sistema tributario pueden no ser suficientes para garantizar que los individuos reconozcan la legitimidad de la autoridad tributaria. La moral tributaria vincula la legitimidad con la credibilidad del Estado, ente del que emana la autoridad-potestad tributaria. La credibilidad se define como la capacidad que tiene un sujeto para hacerse creer, esencia de la legitimidad de la autoridad en el sentido descrito al comienzo de esta sección, y que convierte la coerción en un ejercicio residual del poder (Casinos Mora, 1999). Si el Estado carece de credibilidad, la autoridad tributaria estará desprovista de la legitimidad que le habilita en el uso de la persuasión frente a la coerción. Este aspecto es de enorme relevancia puesto que, implica que dos sistemas tributarios idénticos aplicados sobre sociedades similares darán lugar a niveles distintos de cumplimiento tributario si existen diferencias relevantes en la credibilidad del Estado y de sus instituciones.

Por otro lado, la potestad entendida como coerción es necesaria por cuanto la legitimidad puede variar en el tiempo y depender de las características inherentes al individuo y a la sociedad a la que pertenece. Las características demográficas, socioeconómicas, así como la actitud individual frente al cumplimiento tributario definen una "psicología tributaria" (Schmölders, 1951), que puede favorecer o minar la moral tributaria. De igual modo, los valores colectivos y las normas de cada sociedad pueden tener efectos mensurables sobre la disposición a pagar impuestos (Luttmer, 2014). La confianza en las instituciones, a diferencia de la credibilidad que es objetiva y propia de la autoridad, es subjetiva e inherente a un individuo en su relación con el entorno. Asimismo, una cultura común da forma a las acciones colectivas, de ahí a las instituciones y al desempeño de los gobiernos (Schneider, 2007).

El enfoque de regulación receptiva (responsive regulation, en inglés), sugiere que tanto el poder coercitivo como la confianza son necesarios (Braithwaite, 2007). En una perspectiva dinámica, la educación del contribuyente, el diseño de estrategias destinadas a aumentar la confianza (clima de confianza), y una postura de motivación al compromiso incrementarían la moral tributaria (Kirchler et. al., 2008).

Finalmente, los contribuyentes también pueden sentirse impelidos a contribuir a cambio de bienes y servicios que el Estado puede producir de forma más eficiente que la iniciativa privada (clima de servicios). Los fallos de mercado justifican el papel del Estado como proveedor directo o indirecto de bienes cuando los mercados no satisfacen las condiciones del Primer Teorema del Bienestar. Sin embargo, el rol de corrector de fallos de mercado es una facultad otorgada condicional a la eficiencia, eficacia y efectividad del Estado como proveedor, criterios que directa o indirectamente refuerzan la credibilidad de la autoridad y la confianza del contribuyente, legitimando tanto la autoridad como el uso potestativo del poder.

\section{Factores determinantes de la moral tributaria}

En la sección anterior se han enumerado diferentes factores que contribuyen a la moral tributaria y que pueden ser clasificados en factores sociales y demográficos, factores institucionales y factores culturales. A su vez, los factores institucionales se pueden clasificar en coercitivos y de legitimidad. 
Así, a modo de resumen, el cumplimiento tributario dependería de: a) los costos del incumplimiento (coerción); b) las características psicológicas, personales y culturales (exógenos al sistema tributario); c) el bienestar generado por el uso de impuestos en la producción de bienes públicos (persuasión mediante un clima de servicios); y d) la interacción entre la autoridad y los contribuyentes (persuasión mediante un clima de confianza). Dicha clasificación tiene un significado fuerte para el diseño del sistema tributario.

En términos generales, si el cumplimiento depende principalmente de factores inherentes al individuo, insustanciales a la legitimidad, el ejercicio de la coerción y los costes del incumplimiento son las herramientas más efectivas en el diseño de un sistema impositivo. Sin embargo, si el cumplimiento depende principalmente de variables institucionales y de legitimidad (clima de confianza y servicios), la autoridad tributaria puede diseñar un sistema tributario que mejore el cumplimiento, excluyendo el uso de la coerción como principal herramienta. Entre ambos, si el cumplimiento depende principalmente de las variables culturales, tal vez se pueda diseñar un sistema tributario respaldado por el sistema educativo que podría mejorar la disposición a pagar impuestos a largo plazo.

Respecto a variables demográficas y socioeconómicas, Hofmann (2017) analiza 459 muestras recogidas de 1519 documentos sobre investigación tributaria publicados entre 1958 y 2012, y encuentran un efecto positivo del sexo (femenino) y la edad sobre el cumplimiento tributario, y una relación negativa entre educación e ingresos. Por otro lado, Anderhub (2002) para Alemania, y Alm et. al. (2006) para EE. UU. y 14 países europeos, también hallan una relación positiva entre ser mujer y la moral fiscal, donde ser mujer implica menor tolerancia a la evasión fiscal, o de forma equivalente, una mayor moral fiscal.

En general, Hofmann (2017) encuentra que, aunque las características sociodemográficas se correlacionan significativamente con el cumplimiento tributario, su poder predictivo es limitado para edad y sexo, y es insignificante para la educación y el ingreso. Por otro lado, Prieto et. al. (2006) hallan que características personales no tienen efecto sobre la moral fiscal, con excepción de la variable edad, cuyo parámetro es significativo y con signo negativo: a mayor edad menor tolerancia con el incumplimiento tributario. Un resultado similar obtiene Kirchler (1999) para Austria, puesto que encuentra que los jóvenes son más permisivos con la evasión fiscal. Por otro lado, Torgler (2007) sostiene que el efecto de la educación no está claro, ya que se relaciona con la moral tributaria a través del mejor conocimiento de: (a) reglas tributarias, (b) oportunidades de evasión, y (c) beneficios obtenidos del gasto público. Además, dependiendo del nivel de aversión al riesgo y de la progresividad del impuesto a la renta, la política tributaria puede aumentar o reducir la moral tributaria.

El autoempleo o ejercicio de actividades no dependientes de un empleador tiene una relación negativa sobre la moral tributaria. Los contribuyentes asalariados o dependientes muestran un cumplimiento tributario mayor, debido que los impuestos son deducidos en origen y, por lo tanto, no tienen otra opción que no sea cumplir. En cambio, los contribuyentes no asalariados (empresarios, rentistas, profesionales independientes y trabajadores informales), a menudo, tienen la posibilidad de decidir si cumplen o no con la obligación tributaria (Lago-Peñas y Lago-Peñas, 2010; Saad, 2012).

En relación con factores institucionales, Torgler (2005) obtiene correlaciones estadísticamente significativas y positivas entre la moral fiscal y la calidad de las instituciones, la confianza en el gobierno, la satisfacción con los servicios públicos y la participación. Por ejemplo, Alm et. al. (1993) hallan que los individuos son menos propensos a evadir impuestos cuando se concede al contribuyente el derecho a destinar parte de los impuestos recaudados a una organización benéfica o cultural. Es decir, cuando el contribuyente toma parte en la toma de decisiones del destino de sus impuestos. En resumen, cuanto mayor sea la participación en el proceso de toma de decisiones y mayor la transparencia en el uso de los fondos recaudados, mayor será el cumplimiento tributario.

Otro resultado importante se refiere a la ideología política del individuo, donde ser próximo a partidos de extrema izquierda o derecha reduce la moral fiscal (Prieto et. al., 2006). Este resultado se puede interpretar en términos de legitimidad, en el sentido que individuos con ideologías extremas se sienten menos identificados 
con la legalidad vigente. La actitud ante la tributación no debería necesariamente ser la misma cuando los individuos se consideran integrados en el sistema legal del país, que cuando se ven forzados a acatar reglas con las que no se sienten identificados (Hirshman, 1978). Por otro lado, Persson y Svensson (1989) muestran que gobiernos de ideologías extremas mantienen políticas fiscales inconsistentes, lo que desalentaría el cumplimiento del deber tributario.

Las cuestiones culturales pueden controlarse mediante los efectos nacionales y regionales, la religión y el orgullo nacional (véanse, por ejemplo, Alm y Torgler, 2006; Darmayasa y Aneswari, 2015; Gungor Goksu e Izgi Sahpaz, 2015; Lago-Peñas y Lago-Peñas, 2010). Sin embargo, las políticas que influyen en estos factores a menudo tienen resultados indeseables: la religión es un derecho humano protegido, y la promoción del orgullo nacional fomenta el chauvinismo y la xenofobia. De hecho, algunos de estos factores deben considerarse deterministas y no pueden usarse directamente para mejorar el cumplimiento tributario.

\section{La tributación en América Latina}

Los estudios sobre la tributación en América Latina son unánimes en remarcar que el sistema tributario adolece de problemas estructurales importantes. En comparación con los países desarrollados, la carga tributaria promedio en América Latina es la mitad de la registrada en el promedio de los países europeos ${ }^{[1]}$ de la EU-15, y se sitúa 15 puntos del PIB por debajo del promedio de los países de la OCDE (Jiménez y Podestá, 2016a). Pero, aún más preocupante es que la carga tributaria en la región más pobre del mundo, el África Subsahariana (24,5\% del PIB), es 7 puntos superior a la de América Latina (17,6\% del PIB), lo que la situaba la región en 2009 en el penúltimo lugar a escala mundial (Espada, 2013).

Los principales factores que explican la limitada capacidad recaudatoria en América Latina son: (a) una base imponible reducida debido al gran número de exenciones y deducciones, y la existencia de regímenes fiscales simplificados inadecuados; $y$, (b) el alto nivel de incumplimiento debido a la informalidad, a la evasión y a la criminalidad (OCDE, 2018).

Respecto al diseño del sistema tributario, el amplio uso de exenciones, deducciones, alícuotas reducidas, regímenes especiales, entre otros gastos tributarios, se han esgrimido como adecuado para lograr diferentes objetivos, como la atracción de la inversión extranjera directa, el estímulo al ahorro, el desarrollo de los mercados financieros, de una larga lista de objetivos cuestionables y escasamente evaluados (OCDE, 2018). 
TABLA 1.

América Latina y el Caribe: tasas estimadas de incumplimiento tributario en el Impuesto al Valor Agregado (IVA) y en el Impuesto sobre la Renta (IR)

\begin{tabular}{|c|c|c|c|c|c|c|}
\hline & \multicolumn{3}{|l|}{ IVA } & \multicolumn{3}{|l|}{ IR } \\
\hline & Año & Total & Período & Personas & Sociedades & Total \\
\hline Argentina & 2007 & 0,20 & 2005 & - & - & 0,50 \\
\hline Bolivia & 2004 & 0,29 & - & - & - & - \\
\hline Brasil & & - & $2006-2010$ & 0,38 & - & - \\
\hline Chile & 2014 & 0,22 & 2009 & 0,31 & 0,27 & 0,29 \\
\hline Colombia & 2012 & 0,23 & 2012 & 0,34 & - & - \\
\hline Costa Rica & 2012 & 0,31 & 2012 & 0,68 & 0,54 & 0,64 \\
\hline Ecuador & 2005 & 0,32 & 2005 & 0,65 & 0,58 & 0,64 \\
\hline El Salvador & 2010 & 0,33 & 2005 & 0,51 & 0,36 & 0,45 \\
\hline Guatemala & 2014 & 0,40 & 2006 & 0,63 & 0,70 & 0,64 \\
\hline México & 2012 & 0,24 & 2004 & 0,46 & 0,38 & 0,42 \\
\hline Nicaragua & 2006 & 0,38 & $2006-2010$ & 0,43 & - & - \\
\hline Panamá & 2006 & 0,34 & $2006-2010$ & 0,62 & - & - \\
\hline Paraguay & 2010 & 0,34 & $2006-2010$ & 0,47 & - & - \\
\hline Perú & 2008 & 0,37 & 2006 & 0,51 & 0,33 & 0,49 \\
\hline $\begin{array}{l}\text { R. } \\
\text { Dominicana }\end{array}$ & 2010 & 0,30 & 2009 & 0,69 & 0,52 & - \\
\hline Uruguay & 2012 & 0,13 & $2006-2010$ & 0,48 & - & - \\
\hline
\end{tabular}

Jiménez y Podestá (2016b). Elaborado por los autores.

A pesar de las diferencias metodológicas entre los distintos países para medir su peso, las estimaciones situarían los gastos tributarios en un rango de entre 2 y 7 puntos del PIB, lo que representaría entre el $7 \%$ y el 40\% de la presión fiscal del Impuesto sobre la Renta Personal (Jiménez y Podestá, 2016c); y, en promedio, superarían el 50\% del salario medio de la región. Además, los gastos tributarios aumentan la complejidad en la aplicación normativa tributaria, lo que redundaría en mayores costes de fiscalización y de cumplimiento tributario, creando espacios para interpretaciones ad hoc que favorecerían la evasión y elusión fiscal.

Asimismo, los regímenes de tributación simplificada ampliamente difundidos para el tratamiento de los pequeños contribuyentes, en general, implican una carga tributaria menor. Es decir, la simplificación no es administrativa, sino una forma de incumplimiento tributario tolerado. Además de la falta de control, no existen mecanismos que obliguen a los contribuyentes a tributar por el sistema regular (OCDE, 2018).

Por otro lado, las altas tasas de evasión también limitan la recaudación de ingresos (Tabla 1). Según Jiménez y Podestá (2016b), la tasa de evasión promedio del Impuesto sobre el Valor Agregado (IVA) es del 29\%, y del Impuesto sobre la Renta Personal (IRP) alcanza las tasas promedio del 51\% (Gómez-Sabaíni y Jiménez, 2011; AECID, 2017). Además, menos de un tercio de los impuestos recaudados corresponderían a impuestos directos donde, según Gómez-Sabaíni et. al. (2010), el 60\% (o más) del IRP provendrían de trabajadores asalariados (OCDE, 2018).

Asimismo, las contradicciones del sistema tributario en América Latina son una medida de la falta de una verdadera ingeniería tributaria. Según la OCDE (2018), la recaudación del impuesto sobre la renta de personas naturales y jurídicas en América Latina ${ }^{[2]}$ representa el 1,8\% y el 3\% del PIB, respectivamente, frente al $8,4 \%$ y $2,8 \%$ para la OCDE, y el $10 \%$ y el 2,7\% para la EU. Es decir, la carga tributaria recae sobre todo sobre las empresas, en detrimento de la inversión productiva; y, sobre los asalariados, una fragrante violación del principio de equidad sobre el que se sustenta la legitimidad de cualquier sistema tributario democrático. Asimismo, resulta aparentemente contradictorio que las tasas marginales máximas y mínimas promedio del IRP hayan pasado del 6\% y $47.9 \%$ en 1985 al 10\% y 27\% en 2015, pero que la recaudación aumentara desde el 1,3\% al 1,8\% (Barreix et. al., 2017), una versión perversa de la Curva de Laffer. 
GRÁFICO 1.

Ingresos tributarios totales (\% del PIB) de los países América Latina y Caribe (AL y C), 2016

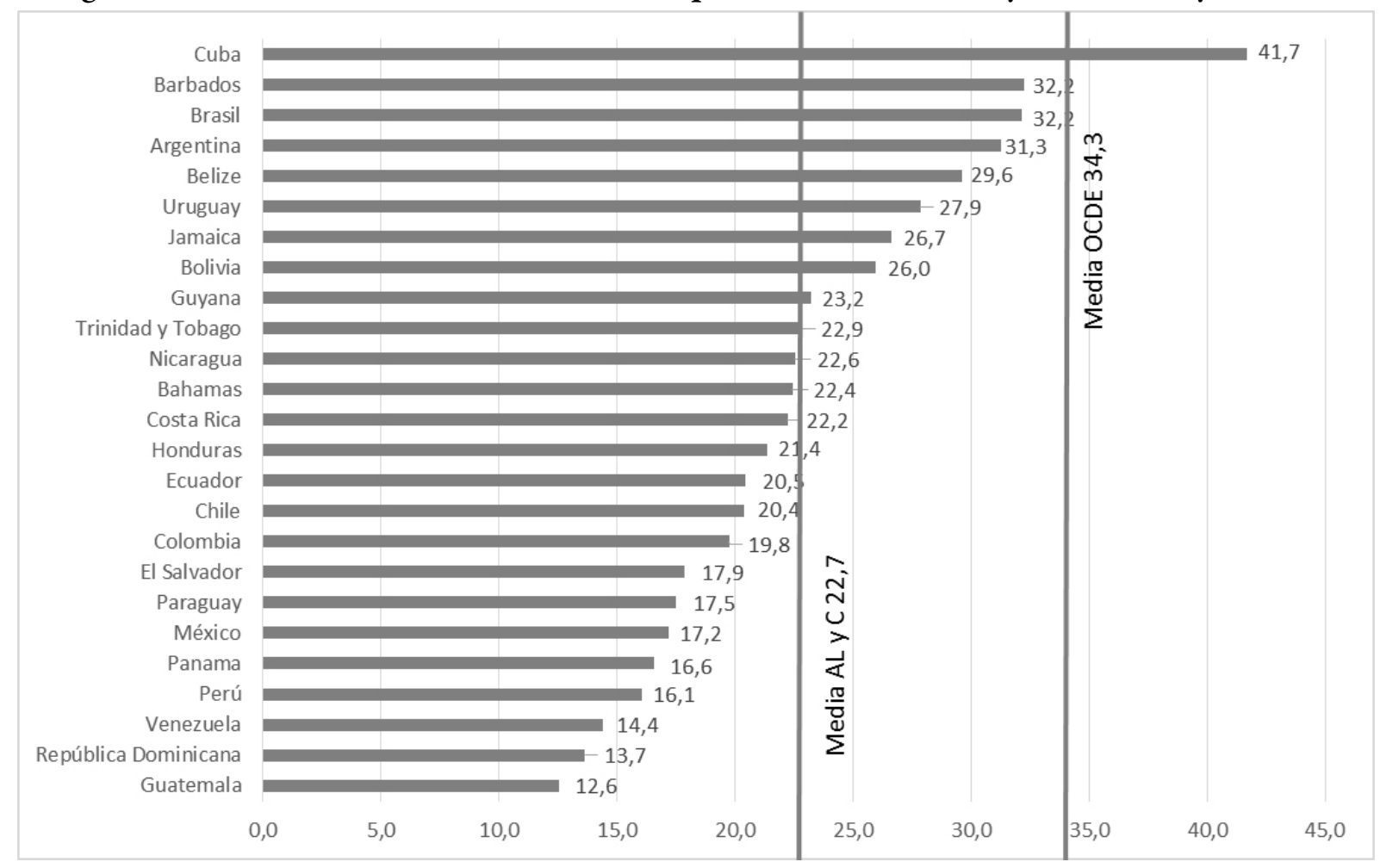

OCDE / CEPAL / CIAT / BID 2018 (OCDE, 2018). Elaborado por los autores.

Nota: Las cifras excluyen los ingresos del gobierno local para Argentina (pero incluyen los ingresos provinciales), Bahamas, Barbados, Cuba, República Dominicana, Honduras y Venezuela, ya que

los datos no están disponibles. La media para OCDE incluye a Chile y México. La media para

América Latina es el promedio no ponderado de los 25 países de América Latina y el Caribe.

Sin embargo, se debe remarcar que las cifras promedio son engañosas con respecto a la verdadera magnitud del problema. Las diferencias entre los países de la región son importantes: la carga tributaria en Argentina y Brasil era superior al 30\% del PIB en 2008, frente a países como México, El Salvador, Guatemala y República Dominicana y Venezuela que no alcanzan el 15\% del PIB (Espada, 2009). Además, los países integrantes de la Commonwealth y Cuba mejoran los valores promedios en las estadísticas para América Latina y Caribe. El Gráfico 1 muestra que, de los 25 países de América Latina y Caribe, sólo 10 países superan la media de ingresos tributarios (11,6 puntos por debajo de la media de la OCDE con Chile y México incluidas), de los cuales cinco son miembros de la Commonwealth of Nations (Barbados, Belice, Guyana, Jamaica y Trinidad y Tobago), y 1 es la socialista Cuba. Por lo tanto, sólo cuatro países propiamente latinos y con economía de mercado (Brasil, Argentina, Uruguay y Bolivia) tienen ingresos fiscales por encima de la media de la región.

\section{Datos y Metodología}

Ante todo, cabe señalar la dificultad de medir la moral fiscal de una sociedad o de un individuo. La moral o ética es algo personal y por lo tanto, su medición adolece de la subjetividad intrínseca al concepto. Habitualmente, se utilizan indicadores agregados de moralidad o inmoralidad fiscal, construidos a partir de encuestas de opinión sobre las actitudes de los ciudadanos ante diferentes aspectos de la vida económica, política y social (Frey W., 1984; Torgler B., 2003). Estos indicadores se utilizan para contrastar las motivaciones socioeconómicas y político-institucionales para la evasión de impuestos. Asimismo, es necesario remarcar que la literatura económica sobre la evasión fiscal está históricamente condicionada por la dificultad 
de medir una actividad que es "ilícita" con evidentes errores de información (ver métodos de cálculo en Jorratt y Podestá, 2010).

En este estudio se utilizan los datos que provienen de la encuesta de opinión Latinobarómetro para el año 2015. Consta de 20250 entrevistas realizadas en 18 países, representativas de la opinión de los latinoamericanos sobre diferentes cuestiones. Los datos son recogidos y documentados anualmente por la Corporación Latinobarómetro, una organización no gubernamental que se encarga de realizar las encuestas y publicar los resultados.

Latinobarómetro goza de independencia y los datos tratan de ser representativos de la realidad nacional de cada país. Realizada cada año, el muestreo es polietápico, estratificado, probabilístico en las primeras fases y por cuotas en la última de extracción, además de proporcional por tamaño del estrato y de la localidad. En la encuesta para 2015, las aproximadamente 1200 observaciones por país se recogen en 4 etapas, con un error muestral de $2.8 \%$, y con una representación del $100 \%$ de la población nacional.

\section{Metodología}

La metodología empleada estima la probabilidad de incumplimiento tributario como función de las variables indicativas de los factores que inciden sobre la moral tributaria. La variable dependiente es la pregunta "Acciones que ha realizado: Rehusar pagar impuestos (P21TGB.F)" cuyas respuestas son "nunca”, "podría”, "ha realizado".

Se asume que los individuos que declaran que "han rehusado a pagar impuestos" son los que incumplen con su deber tributario. Esta interpretación es benévola, puesto que asume que los individuos que podrían rehusar también cumplen con su deber tributario. De hecho, en la muestra sólo el 4,5\% declaran haber rehusado pagar impuestos, una cifra muy lejana a las tasas de incumplimiento tributario para la zona. Sin embargo, esta simplificación facilita ver con claridad qué factores inciden sobre el incumplimiento tributario declarado (y no supuesto).

Por otro lado, el modelo de análisis Probit es un procedimiento de estimación muy intuitivo: supongamos que la inmoralidad tributaria, que se denotará por $Y^{*}$, se puede explicar por la relación lineal, $Y^{*}=X \beta+\varepsilon$, donde $X$ es la matriz $I \times J$ que contiene las $J$ variables explicativas observadas para los I individuos, y $\varepsilon$ es el término de error. Aunque la inmoralidad $Y^{*}$ es inobservable, se puede observar una variable dicotómica que toma el valor $Y=1$ si el individuo rehúsa pagar impuestos y, $Y=0$, en caso contrario.

Consideremos que la probabilidad de incumplimiento es igual a la probabilidad que el individuo rehúse pagar impuestos la cual, a su vez, es igual a la probabilidad de que la inmoralidad $\mathrm{Y}^{*}$ sea positiva, entonces, $P(Y=1)=P\left(Y^{*}>0\right)=P(X \beta+\varepsilon>0)$. Asumiendo que $\varepsilon$ es una variable aleatoria continua simétrica, se satisface que, $P(Y=1)=P\left(Y^{*}>0\right)=P(\varepsilon>-X \beta)=P(\varepsilon \leq-X \beta)=F(X \beta)$, donde $F$ es la función de distribución de $\varepsilon$. Dado este resultado general, el modelo Probit estima el vector $\beta$ de $J$ parámetros hallando el máximo de la función de verosimilitud, bajo la hipótesis que $\varepsilon$ tiene distribución normal, es decir, que $F$ es la función de distribución normal (Maddala, 1983).

Los $J$ parámetros estimados $b_{j}$ del parámetro $\beta_{j}$ se interpretan en términos del aumento o disminución en la probabilidad de incumplimiento como consecuencia de un incremento en la variable $\mathbf{x}_{j}$ (marginal, si continua, y unitario, si discreta). Por lo tanto, siempre que $\beta_{j}$ sea significativamente diferente de cero, se interpreta que un incremento en la variable $x_{j}$ aumenta la probabilidad de incumplimiento cuando $b_{j}>0$; y, que disminuye esta probabilidad si $b_{j}<0$,. Observe que se interpreta el signo del coeficiente, pero no la magnitud. 
TABLA 2.

Variables explicativas y tipo

\begin{tabular}{|l|l|l|}
\hline Variable & Explicación (código variable) & Tipo \\
\hline Masculino & Sexo del entrevistado (S12) & Dicotómica \\
\hline Edad & Edad del entrevistado (S13) & Continua \\
\hline Clase social & Clase social subjetiva (S6) & Ordinal \\
\hline Soltero & $\begin{array}{l}\text { Recodificación de Estado civil del entrevistado } \\
\text { (S11) }\end{array}$ & Dicotómica \\
\hline Educación & Educación del entrevistado (S19) & Ordinal \\
\hline Educación progenitores & Valores ajustados por MCO (S20 sobre S4 y S19) & Continua \\
\hline Dependencia & Situación laboral del entrevistado (S21A) & Ordinal \\
\hline Ideología & Ideología Política del entrevistado (P27ST) & Ordinal \\
\hline Confianza sociedad & $\begin{array}{l}\text { Indicador sintético construido con ACM (ver } \\
\text { Tabla 3) }\end{array}$ & Continua \\
\hline Confianza instituciones & $\begin{array}{l}\text { Indicador sintético construido con ACM (ver } \\
\text { Tabla 3) }\end{array}$ & Continua \\
\hline Confianza Estado & Confianza en el Estado (P19ST.F) & Ordinal \\
\hline $\begin{array}{l}\text { Tolerancia con } \\
\text { incumplimiento }\end{array}$ & Escala de justificación: Evadir impuestos (P56ST) & Ordinal \\
\hline Adhesión gobierno & Adhesión Política del entrevistado (P23TGBS.A) & Ordinal \\
\hline Satisfacción hospitales & Satisfacción con los Hospitales Públicos (P53ST.A) & Ordinal \\
\hline $\begin{array}{l}\text { Satisfacción educación } \\
\text { pública }\end{array}$ & Satisfacción con la Educación Pública (P53ST.B) & Ordinal \\
\hline Satisfacción policía & Satisfacción con la Policía (P53ST.C) & Ordinal \\
\hline Satisfacción justicia & Satisfacción con el Sistema Judicial (P53ST.D) & Ordinal \\
\hline Cono Sur & Argentina, Chile, Uruguay (IDENPA) & Dicotómica \\
\hline Brasil & Brasil (IDENPA) & Dicotómica \\
\hline México & México (IDENPA) & Dicotómica \\
\hline $\begin{array}{l}\text { Centro América y Caribe } \\
\text { Costa Rica, Rep. Dominicana, El Salvador', } \\
\text { Guatemala, Honduras, Nicaragua (IDENPA) }\end{array}$ & Dicotómica \\
\hline Otros & Panamá, Paraguay (IDENPA) & Dicotómica \\
\hline
\end{tabular}

Latinbarómetro 2016. Elaborado por los autores.

En el caso de la variable "Educación de los progenitores", los datos ausentes representan el $11.45 \%$ de los casos. Es decir, si bien la educación tributaria recibida en el hogar es importante, la inclusión de esta variable podría sesgar los resultados a favor de los que sí declaran la educación de los progenitores, en contra de los que no declaran esta información. Para resolver este problema, se construye una variable sintética donde los valores ausentes se sustituyen por los valores predichos o ajustados por el modelo MCO, donde se regresa la variable nivel educativo de los padres (S20) sobre las variables ingreso subjetivo (S4) y nivel educativo del entrevistado (S19).

En el caso de las variables relacionadas con la confianza, la información está excesivamente discriminada para grupos específicos. Es decir, si se tratara de hallar el efecto de la confianza en grupos concretos (sindicatos, estudiantes, institución electoral, etc.), seguramente se encontraría que ninguna de ellas contribuye mucho a la moral tributaria. Sin embargo, la confianza en grupos homogéneos como sociedad e instituciones puede dar una respuesta más satisfactoria. La cuestión relevante es cómo construir un indicador óptimo de confianza en estos grupos.

El análisis de correspondencia múltiple $(\mathrm{ACM})$ es una extensión del análisis de correspondencia que permite analizar el patrón de relaciones y es adecuado para reducir de forma óptima la dimensión de una matriz de datos categóricos relacionados. Por ese motivo, se utilizará el ACM para construir variables sintéticas indicativas de la confianza. Como tal, el ACM también se puede ver como una generalización del análisis de componentes principales cuando las variables que se analizan son categóricas y por ello, en lugar de distancias Mahalanobis, se debe utilizar distancias Ji-Cuadrado. 
La Tabla 3 muestra las variables activas utilizadas para construir los indicadores y los valores de la $\alpha$ de Cronbach y la inercia de la primera dimensión, utilizadas para medir la fiabilidad y la calidad de la representación, respectivamente (Greenacre y Blasius, 2006; Greenacre,2017).

TABLA 3.

Variables activas y medidas de fiabilidad y calidad de la representación

\begin{tabular}{|l|l|l|l|}
\hline Indicador & Variables activas & $\begin{array}{l}\alpha \text { de } \\
\text { Cronbach }\end{array}$ & Inercia \\
\hline $\begin{array}{l}\text { Confianza } \\
\text { sociedad }\end{array}$ & $\begin{array}{l}\text { Confianza en sindicatos (P19ST.A), Estudiantes } \\
\text { (P19ST.B), Medios de Comunicación (P19ST.D), } \\
\text { Empresas privadas (P19ST.E) }\end{array}$ & 0.659 & 0.494 \\
\hline $\begin{array}{l}\text { Confianza } \\
\text { instituciones }\end{array}$ & $\begin{array}{l}\text { Confianza en partidos políticos (P19ST.C), } \\
\text { Bancos (P19ST.G), Institución electoral (P19N.H) }\end{array}$ & 0.699 & 0.602 \\
\hline
\end{tabular}

Elaborado por los autores.

Finalmente, se comprueba la buena especificación del modelo Probit contrastando la hipótesis que la perturbación se distribuye según una normal esférica (homocedástica y no autocorrelacionada).

\section{RESULTADOS Y DISCUSIÓN}

La Tabla 4 muestra el modelo Probit estimado, donde no se puede rechazar la hipótesis nula que las perturbaciones son homocedásticas, no autocorrelacionadas y que proceden de una distribución normal, con lo cual la especificación es adecuada. 
TABLA 4.

Factores que inciden sobre el cumplimiento tributario estimado con un modelo Probit

\begin{tabular}{|c|c|c|c|c|c|}
\hline Variable & $\mathrm{b}_{\mathrm{j}}$ & $\operatorname{std}\left(b_{j}\right)$ & $z$ & p-value & \\
\hline Constante & $-2,32710$ & 0,27025 & $-8,612$ & 0 & $* * *$ \\
\hline Masculino & 0,06616 & 0,03731 & 1,773 & 0,0762 & $*$ \\
\hline Edad (log) & 0,08167 & 0,02642 & 3,092 & 0,002 & $* * *$ \\
\hline Clase social & 0,05930 & 0,02070 & 2,865 & 0,0042 & $* * *$ \\
\hline Soltero & 0,02869 & 0,02106 & 1,362 & 0,1732 & \\
\hline Educación & $-0,00832$ & 0,00555 & $-1,499$ & 0,1339 & \\
\hline Educación progenitores & 0,01008 & 0,00516 & 1,956 & 0,0505 & * \\
\hline Dependencia & $-0,04864$ & 0,01924 & $-2,528$ & 0,0115 & ** \\
\hline $\begin{array}{l}\text { Ideología izquierda- } \\
\text { derecha (log) }\end{array}$ & $-0,07862$ & 0,01372 & $-5,732$ & 0 & $* * *$ \\
\hline Confianza sociedad & $-0,05793$ & 0,02670 & $-2,146$ & 0,0319 & ** \\
\hline Confianza instituciones & 0,10099 & 0,03002 & 3,364 & 0,0008 & $* * *$ \\
\hline $\begin{array}{l}\text { Confianza instituciones } \\
\text { (sq) }\end{array}$ & 0,04218 & 0,01424 & 2,962 & 0,0031 & $* * *$ \\
\hline Confianza Estado & 0,03888 & 0,02829 & 1,374 & 0,1694 & \\
\hline Satisfacción hospitales & 0,04262 & 0,02547 & 1,673 & 0,0942 & * \\
\hline Adhesión al gobierno & 0,02286 & 0,01423 & 1,607 & 0,1082 & \\
\hline $\begin{array}{l}\text { Satisfacción educación } \\
\text { pública. }\end{array}$ & $-0,09999$ & 0,02569 & $-3,892$ & 0 & $* * *$ \\
\hline Satisfacción hospitales & 0,04262 & 0,02547 & 1,673 & 0,0942 & $*$ \\
\hline Satisfacción policia (log) & 0,00982 & 0,02708 & 0,363 & 0,7168 & \\
\hline Satisfacción justicia & 0,02987 & 0,02845 & 1,050 & 0,2937 & \\
\hline $\begin{array}{l}\text { Tolerancia con } \\
\text { incumplimiento }\end{array}$ & 0,03987 & 0,00655 & 6,087 & 0 & $* * *$ \\
\hline Cono Sur & $-0,23345$ & 0,06307 & $-3,702$ & 0,0002 & $* * *$ \\
\hline Brasil & $-0,03554$ & 0,08749 & $-0,406$ & 0,6846 & \\
\hline México & 0,11742 & 0,0758 & 1,549 & 0,1213 & \\
\hline Centro América y Caribe & 0,12397 & 0,04863 & 2,549 & 0,0108 & ** \\
\hline Otros (excepto CAN) & 0,03060 & 0,06438 & 0,475 & 0,6347 & \\
\hline
\end{tabular}

Encuesta Latinobarómetro 2015. Elaborado por los autores.

Variable dependiente: Rehusar pagar impuestos. Número de observaciones válidas: 15160 . Errores estándar robustos $(\mathrm{Std}){ }^{* * *} \mathrm{p}<0,01,{ }^{* *} \mathrm{p}<0,05,{ }^{*} \mathrm{p}<0,1$

\section{Efecto de variables demográficas y socio económicas}

Con respecto al sexo, no se puede rechazar la hipótesis nula que el parámetro es cero para un nivel de confianza del $5 \%$. Dicho de otro modo, hay evidencia a favor de la hipótesis de que no existen diferencias por sexo en la probabilidad de incumplimiento, coherente con los resultados obtenidos por Prieto et. al. (2006) y que no contradicen los resultados obtenidos por Hofmann (2017), Anderhub (2002) y Alm et. al. (2006).

En el caso de la edad, el logaritmo de la variable corrige la heterocedasticidad, lo que apunta hacia la hipótesis que existe una componente de aprendizaje en el incumplimiento tributario. El parámetro es significativamente diferente de cero y con signo positivo. Es decir, en América Latina, la edad aumenta la probabilidad de incumplimiento tributario, aunque este incremento disminuye a medida que el individuo envejece. Por lo tanto, aunque el signo es el contrario al esperado (Hofmann, 2017; Kirchler, 1999), la edad tiene un efecto positivo decreciente. Este resultado puede deberse a que, aunque la conciencia tributaria pueda aumentar con la edad, no hay incentivos para financiar servicios públicos e infraestructuras cuyo disfrute disminuye con la edad.

La clase social subjetiva tiene el parámetro significativamente diferente de cero y positivo, evidencia de que el ascenso social subjetivo aumenta la probabilidad de incumplimiento tributario (Hofmann, 2017). Es 
decir, si se considera que la clase social subjetiva es una variable proxi de la renta subjetiva, se halla que la renta subjetiva aumenta el incumplimiento tributario. Es un resultado interesante puesto que, el incremento en la probabilidad de incumplimiento ocurre si el individuo cree que está en una posición social mejor, aunque eso no sea cierto. De hecho, según el Latinobarómetro, sólo el 22,3\% de los encuestados se creen de clase baja, lo que contrasta con los informes que muestran que América Latina es la región más desigual del mundo (De Ferranti et. al., 2004).

Respecto al estado civil (soltero), no se puede rechazar la hipótesis nula que el parámetro es cero para un nivel de significación del 5\%. Por lo tanto, en América Latina, hay evidencia de que las cargas familiares no inciden sobre la moral tributaria (Prieto et. al., 2006).

Por otro lado, hay evidencia de que individuos con posturas de izquierdas son más cumplidores que los individuos con posturas ideológicas cercanas a derecha política, aunque no se ha encontrado el efecto de posturas extremas discutido en la Sección: Factores determinantes de la moral tributaria. Más bien, se halló que el incremento en la probabilidad de incumplimiento se hace más suave a medida que el individuo se acerca a posturas de extrema derecha. Esa discrepancia puede deberse a cualquiera de los siguientes motivos: a) el efecto de posturas extremas no existe en América Latina; b) los individuos con posturas extremas rechazan realizar la encuesta; o, c) no existen posturas extremas en el sentido definido por Prieto et. al. (2006).

En cuanto a la educación del entrevistado, no se puede rechazar la hipótesis nula que el parámetro es cero. Por lo tanto, no hay evidencia de que la educación del individuo aumente la probabilidad de incumplimiento, consistente con la hipótesis que los efectos negativos y positivos de la educación se compensan (Torgler, 2007). Sin embargo, se rechaza la hipótesis nula para la educación de los progenitores. Así, la educación tributaria durante la infancia, cuando la educación de los progenitores es más importante, parece ser más relevante que el nivel educativo del individuo. Este efecto no se había estudiado en trabajos similares.

Por otro lado, el nivel de dependencia de los ingresos de un empleador (asalariado frente a cuenta propia o empresarios) favorece el cumplimiento tributario, a menudo, no voluntario (Lago-Peñas y Lago-Peñas, 2010; Saad, 2012; Hofmann, 2017).

Por lo tanto, pese a los matices, los resultados obtenidos para las variables demográficas y socio económicas son coherentes con los obtenidos en estudios similares para otros países y regiones, excepto para la edad (ver Sección: Factores determinantes de la moral tributaria destinada estudiar los factores determinantes de la moral tributaria).

Efecto de variables indicativas de clima de servicios y clima de confianza

Tal como se discute en la Sección: Factores determinantes de la moral tributaria, las variables asociadas a la satisfacción (clima de servicios) y la credibilidad (clima de confianza) de los individuos en las instituciones son indicativas de que es posible diseñar una política tributaria no coercitiva, sustentada sobre la legitimidad del acto, que persuada a los individuos a contribuir voluntariamente (Bergman, 2003; Kirchler et al., 2008; Torgler, 2005).

Con respecto a la confianza en la sociedad, el parámetro es significativamente diferente de cero y negativo, por lo que, cuanto mayor es el clima de confianza generado por la sociedad civil, mayor será la moral tributaria. Este resultado indica que la confianza en la sociedad es un factor que legitima el acto tributario. La intuición detrás de este resultado es que los impuestos son, de hecho, contribuciones a un gasto común en beneficio de la sociedad.

Por otro lado, hay evidencia de que la probabilidad de incumplimiento es una función convexa de la confianza en las instituciones. Dado que la probabilidad estimada en el mínimo de la función es negativa, la confianza en las instituciones ${ }^{[3]}$ (partidos políticos, bancos e institución electoral) aumentaría la probabilidad de incumplimiento, y de forma creciente. Una posible explicación para esta sorprendente relación positiva entre incumplimiento y confianza en las instituciones es que hay una especie de "efecto polizón", entendiendo por tal, que los individuos perciben que no necesitan contribuir tanto cuando las instituciones son confiables. 
Por otro lado, no se rechaza la hipótesis nula que el parámetro asociado a la confianza en el Estado es cero. Así, aunque parezca sorprendente, la confianza en el ente del que emana la potestad-autoridad tributaria no aumenta ni disminuye la probabilidad de incumplimiento. Asimismo, hay evidencia de que la probabilidad de incumplimiento no depende de la adhesión al gobierno, lo que puede ser una buena noticia si se interpreta como que la moral tributaria es ajena a la política; pero, una mala noticia si se tiene en cuenta que eso reduce el número de instrumentos de legitimidad para diseñar la política tributaria.

El grupo de variables relativas a satisfacción se asocian al clima de servicio que genera la provisión de bienes públicos con los impuestos recaudados. De igual modo que con las variables de confianza, el clima de servicio es un factor de legitimidad y, por lo tanto, se espera que disminuya la probabilidad de incumplimiento. En los casos de las variables satisfacción con la policía, la justicia y los hospitales, no se puede rechazar la hipótesis nula que los parámetros asociados a estas variables son cero para un nivel de significación del $5 \%$.

La satisfacción con los hospitales es significativa, pero sólo si se aumenta el nivel de significación al 10\%. En este caso, el parámetro es positivo y no negativo, tal como se espera. Por lo tanto, la satisfacción los servicios hospitalarios aumenta la probabilidad de incumplimiento tributario. Este resultado puede deberse a que la sanidad de pago se encuentra muy extendida entre la población, de modo que los latinoamericanos perciben que pagan dos veces por el mismo servicio. Otra conjetura sobre este resultado es que los individuos no perciben que el incremento en el gasto ${ }^{[4]}$ mejore las prestaciones relativamente a la asistencia privada.

Sólo la satisfacción con la educación es indudablemente significativa y tiene el signo esperado, negativo, indicando que la provisión de educación con fondos públicos reduce la probabilidad de incumplimiento tributario. Ese resultado se debe a que la educación genera externalidades más observables que la salud. Por otro lado, el efecto de doble pago por un mismo servicio desaparece por cuanto no es factible que un individuo se matricule simultáneamente en la educación privada y pública para cursar la misma formación oficial.

En general, las variables más directamente relacionadas con las teorías de moral fiscal y que dan lugar a políticas tributarias sustentadas sobre la legitimidad (persuasión) más que en la coerción (disuasión), se ajustan parcialmente a las teorías expuestas en la Sección: Factores determinantes de la moral tributaria. Así, aunque el clima de confianza generado por la sociedad y las instituciones es relevante, la confianza en las instituciones disminuye la moral fiscal, un resultado sorprendente. $Y$, en caso del clima de servicios, sólo el gasto en educación tiene un significativo efecto positivo sobre la moral tributaria.

\section{Efecto de variables indicativas de diferencias culturales}

No se deber esperar diferencias culturales entre los países de América Latina, por eso, la hipótesis nula es que los parámetros asociados a áreas o países son cero. Sin embargo, si existen diferencias en la cultura tributaria, entonces se podría esperar que algunos de los parámetros fueran significativamente diferentes de cero. Para realizar el contraste, se han agrupado los países: CAN (Ecuador, Bolivia, Perú, Venezuela y Colombia), Cono Sur (Argentina, Chile y Uruguay), Centro América y Caribe (Costa Rica, República Dominicana, El Salvador, Guatemala, Honduras y Nicaragua) y, Otros (Paraguay y Panamá). Además, se han creado las variables Brasil y México, para analizar estos países por separado, dada la complejidad de sus economías. Finalmente, se deja a CAN como variable de referencia. No se rechaza la hipótesis nula de que no existen diferencias culturales entre la CAN, México, Brasil y Otros. En cambio, los parámetros asociados a Cono Sur y Centro América y Caribe son significativamente diferente de cero.

El signo asociado a Cono Sur es negativo, lo que significa que un contribuyente que tributa en cualquiera de los países de esta zona tendrá una probabilidad menor de incumplimiento tributario con respecto a la CAN. Lo contrario ocurre en el caso de los países de Centro América y Caribe cuyos sistemas tributarios no favorecen el cumplimiento tributario con respecto a los países de la CAN. Por lo tanto, existen diferencias culturales con respecto a la moral tributaria, posiblemente provocadas por la misma forma de gestionar los impuestos, tal como se discute en la Sección: Factores determinantes de la moral tributaria.

Finalmente, cabe comentar que no se hallaron diferencias significativas en la probabilidad de incumplimiento en Paraguay y Panamá (Otros) con respecto a la CAN, lo cual es aparentemente 
sorprendente, pero razonable si se tiene en cuenta que es difícil percibir el incumplimiento en un sistema tributario tolerante con el incumplimiento.

\section{Conclusiones}

En este trabajo, se asimila la probabilidad de rehusar pagar impuestos con la probabilidad de incumplimiento tributario. Como tal, esta probabilidad se debe entender como una propensión al incumplimiento tributario, la cual no es equivalente a la probabilidad que el individuo realice actos detectables de incumplimiento tributario. Así, la probabilidad de incumplimiento difiere de la probabilidad que se puede obtener de los actos tributarios observados por una autoridad tributaria. En primer lugar, porque para observar el acto tributario es necesario que este exista, lo cual depende de la norma tributaria. En segundo lugar, porque la probabilidad de observar un acto tributario depende de la probabilidad de detección la cual, en general, es inferior a uno.

Por lo tanto, se podría esperar que trabajos que utilicen datos relativos a la detección de actos tributarios fraudulentos o incorrectos difieran de los resultados obtenidos en este trabajo, es decir, cuando la variable dependiente es el incumplimiento condicionado a que el individuo tiene una deuda tributaria y además, que la autoridad tributaria discrimina correctamente entre "cumplidores" y "no cumplidores". Tal variable excluiría a los individuos sin obligaciones tributarias, aunque este hecho no exime el individuo de tener moral tributaria. Asimismo, sesgaría el estudio en favor de los contribuyentes mejor controlados como los dependientes. En cambio, la probabilidad de incumplimiento se construye a partir de la decisión de rehusar pagar impuestos, inclusive cuando tal obligación no existe, revelando una auténtica moral tributaria.

En cuanto a resultados, en muchos aspectos los resultados obtenidos en este estudio se corresponden a los encontrados para otros países. Así, no se puede rechazar que existen factores de legitimidad que justifican políticas tributarias no coercitivas. El clima de servicios generado por el gasto en la educación favorece la moral tributaria, de igual modo que la confianza en la sociedad. A la vez, tampoco se puede rechazar que la significatividad de los factores socio demográficos, los cuales justifican la necesidad de políticas tributaria coercitivas. Así, aunque la disuasión es necesaria, no es suficiente para garantizar el cumplimiento tributario (Saad, 2012; Braithwaite, 2007).

Sin embargo, muchos de los resultados son poco alentadores, como el hecho de que los poderes del Estado y el Estado mismo no sean entendidos por los individuos como factores de legitimidad tributaria. En particular, es preocupante el hecho que la confianza en las instituciones aumente la probabilidad de incumplimiento.

Este resultado da lugar a que se formule la conjetura de si los latinoamericanos poseen el mismo concepto de confianza manejado por la literatura en tributación, entendiendo por tal la buena fe que se pone en alguien o algo. En tal caso, confianza es la creencia subjetiva directamente relacionada con la credibilidad objetiva que inspiran las instituciones. Por el contrario, si los latinoamericanos entienden por confianza la esperanza de conseguir lo deseado (muchos servicios y pocos impuestos), entonces credibilidad y confianza tienen un sentido diferente al definido por Schneider (2007). Así, bajo la segunda acepción, es un resultado justificable que la confianza en las instituciones aumente la probabilidad de incumplimiento tributario. Sin embargo, esta pregunta devuelve la teoría de la tributación a la sociología, a la psicología y la introduce en la semiología. Posiblemente, la respuesta se encuentre en que los que pueden y deben pagar impuestos son pocos y a menudo, coinciden con la potestad-autoridad tributaria, lo que puede incidir negativamente sobre la legitimidad del acto.

La buena especificación y la representatividad de la muestra garantizan las buenas propiedades asintóticas de los estimadores. Obviamente, este estudio es mejorable, ya que datos más precisos que incluyeran de forma periódica cuestiones relativas a la tributación permitirían obtener resultados mejores. Asimismo, desafortunadamente, no es posible realizar un análisis dinámico entre moral tributaria y sus factores determinantes debido a que Latinobarómetro no incluye esta pregunta de forma periódica. 
Por otro lado, hay una cuestión de fondo poco discutida en la literatura: el modelo Probit se estima asumiendo que la probabilidad individual de incumplimiento es constante. En tal caso, la hipótesis de normalidad es correcta. Pero, si la probabilidad de incumplimiento individual es una variable aleatoria, entonces, la normalidad podría ser un supuesto incorrecto. En particular, si las decisiones individuales dependen de la moral colectiva, bajo intercambiabilidad de las decisiones, el supuesto más adecuado es que la probabilidad de incumplimiento converja casi seguro a una distribución beta, donde la normalidad sería el caso extremo de independencia entre moral individual y moral colectiva (Espinosa y Horna, 2019). Desafortunadamente, los datos disponibles para América Latina no permiten estimar el modelo bajo la hipótesis que la probabilidad de incumplimiento se distribuye según una beta con parámetros desconocidos.

\section{Agradecimientos}

Los autores agradecen a Julio Medina, Marco Naranjo y Yasmín Salazar por sus valiosos comentarios. Esta investigación fue apoyada por la Escuela Politécnica Nacional en Ecuador, Proyecto de Investigación PIIDM-2017-04.

\section{Referencias bibliográficas}

Allingham, M. y Sandmo, A. (1972). Income tax evasion: A theoretical analysis. Journal of Public Economics, 1(3-4), 323-338. doi: 10.1016/0047-2727(72)90010-2.

Alm, J. B., y Torgler, B. (2006). Culture differences and tax morale in the United States and in Europe. Journal of Economic Psychology, 27(2), 224-246. doi: 10.1016/j.joep.2005.09.002.

Alm, J. B. (1993). Fiscal Exchange, Collective Decision Institutions, and Tax Compliance. Journal of Economic Behavior \& Organization, 22(3), 285-303.

Anderhub, V. S. (2002). Tax evasion with earned income an experimental study. FinanzArchiv, 58(2), 188-206.

Barreix, A., J. Benítez and M. Pecho (2017), "Revisiting personal income tax in Latin America: Evolution and impact", OECD Development Centre Working Papers, No. 338, OECD Publishing, Paris, Francia. doi: $10.1787 / 16 d 42 b 4 a-e n$.

Bergman, M. S. (2003). Tax Reforms and Tax Compliance: The Divergent Paths of Chile and Argentina. Journal of Latin American Studies, 35(3), 593-624.

Braithwaite, V. (2007). Responsive regulation and taxation: Introduction. Law \& Policy, 29(1), 3-10. doi: 10.1111/ j.1467-9930.2007.00242.x

Casinos Mora y Francisco Javier (1999). El dualismo autoridad-potestad como fundamento de la organización y del pensamiento político en Roma. Polis: Revista de ideas y formas políticas de la Antigüedad, 11, 85-109.

CEPAL (2017). Panorama Fiscal de América Latina y el Caribe 2017: la movilización de recursospara el financiamiento del desarrollo sostenible. Santiago de Chile, Chile: Comisión Económica para América Latina y el Caribe (CEPAL).

Darmayasa, I., y Aneswari, Y. (2015). The ethical practice of tax consultant based on local culture. Procedia-Social and Behavioral Sciences, 211, 142-148. doi: 10.1016/j.sbspro.2015.11.021.

De Ferranti, David; Ferreira, Francisco H. G.; Perry, Guillermo E.; Walton, Michael. 2004. Inequality in Latin America: breaking with history? Washington, DC, Estados Unidos de América: World Bank Latin American and Caribbean Studies.

Espada, M. V. (2013). Los sistemas tributarios en América Latina. Desafios para la imposición sobre la renta. Madrid, España: ILES-CEPAL.

Alexandra M. Espinosa y Luís Horna (2019) The statistical properties of the threshold model and the feedback leadership condition, Journal of Applied Statistics (online). doi: 10.1080/02664763.2019.1658728

Frey, W. (1984). The hidden economy as an 'unobserved' variable. European Economic Review, 26(1-2), 33-53. 
Gómez Sabaini J. C., J. P. Jiménez y A. Podestá (2010), “Tributación, evasión y equidad en América Latina y el Caribe”. En Juan Pablo Jiménez, J. C. Gómez Sabaini and Andrea Podestá (Ed.), Evasión y equidad en América Latina (pág. 11-65). Santiago de Chile, Chile: Comisión Económica para América Latina y el Caribe (CEPAL).

Greenacre, M. (2017). Correspondence Analysis in Practice, Third Edition. Boca Raton, Estados Unidos de América: Chapman \& Hall/CRC Interdisciplinary Statistics. CRC Press.

Greenacre, M., y Blasius, J. (2006). Multiple Correspondence Analysis and Related Methods. Boca Raton, Estados Unidos de América: Chapman \& Hall/CRC Statistics in the Social and Behavioral Sciences. CRC Press.

Gungor Goksu, G., y K. Izgi Sahpaz (2015). Comparison of Tax Morale of Turkish and Spanish Higher Education Students: The Samples of Sakarya University and the University of Zaragoza. Procedia-Social and Behavioral Sciences, 186, 222-230. doi: 10.1016/j.sbspro.2015.04.027.

Hirshman, A. (1978). Exit, voice, and loyalty. Cambridge, Reino Unido: Harvard University Press.

Hofmann, M. V. (2017). Tax compliance across sociodemographic categories: Metaanalyses of survey studies in 111 countries. Journal of Economic Psychology, 62, 63-71.

Jiménez, J.P. y A. Podestá (2016a), “La tributación sobre la renta en América Latina: desafíos y perspectivas”, En Juan C. Gómez Sabaini, Juan Pablo Jiménez, Ricardo Martner (Ed.), Consensos y Conflictos en la política tributaria de América Latina (pág. 67-91). Santiago de Chile, Chile: Comisión Económica para América Latina y el Caribe (CEPAL).

Jiménez, J. P. y A. Podestá (2016b), "Situación económica y social en América Latina. Ingresos tributarios y carga fiscal” En Agencia Española para la Cooperación Internacional y el Desarrollo (Ed.), Los sistemas tributarios en América Latina (pág. 21-23). Madrid, España: Agencia Española para la Cooperación Internacional y el Desarrollo (AECID).

Jiménez, J.P. y A. Podestá (2016c), "Los impuestos sobre la renta de las personas físicas en América Latina”, En Agencia Española para la Cooperación Internacional y el Desarrollo (Ed.), Los sistemas tributarios en América Latina (pág. 21-23). Madrid, España: Agencia Española para la Cooperación Internacional y el Desarrollo (AECID).

Jorratt, M. y Andrea Podestá (2010). “Análisis comparativo de las metodologias empleadaspara la estimación de la evasión en el impuesto a la renta”. En Juan Pablo Jiménez, Juan Carlos Gómez Sabaini y Andrea Podestá (Coordinadores), Evasión y equidad en América Latina (pág. 69-94). Santiago de Chile, Chile: Comisión Económica para América Latina y el Caribe (CEPAL).

Kirchler, E. H. (1999). Reactance to taxation: employers' attitudes towards taxes. Journal of Socio-Economics, 28(2), 131-138.

Kirchler, E. H. (2008). Enforced versus voluntary tax compliance: The "slippery slope" framework. Journal of Economic Psychology, 29(2), 210-225.

Kountouris, Y. y K. Remoundou (2013). Is There a Cultural Component in Tax Morale? Evidence from Immigrants in Europe. Journal of Economic Behavior \& Organization, 96, 104-119.

Lago-Peñas, I y Lago-Peñas S. (2010). The determinants of tax morale in comparative perspective: Evidence from European countries. European Journal of Political Economy, 26(4), 441-453.

Luttmer, E. F. (2014). Tax morale. The Journal of Economic Perspectives, 28(4), 149-168.

Maddala, G. S., (1986), Limited-Dependent and Qualitative Variables in Econometrics. Cambridge: Cambridge University Press.

OCDE, et al. (2018), Estadísticas tributarias en América Latina y el Caribe 2018. Paris, Francia: OECD Publishing. doi: 10.1787/rev_lat_car-2018-en-fr.

Pecho, M., F. Peláez y J. Sánchez (2012), “Estimation of tax non-compliance in Latin America 2000-2010”, Documento de Trabajo, 2012/3, Centro Interamericano de Administraciones Tributarias (CIAT), Cuidad de Panamá, Panamá.

Persson, T.y L.E. O. Svensson (1989). Why a stubborn conservative would run a deficit: Policy with time-inconsistent preferences. The Quarterly Journal of Economics, 104(2), 325-345. 
Prieto Rodriguez, J., y Sanzo Pérez, M. J. (2006). Análisis económico de la actitud hacia el fraude fiscal en España. Hacienda Pública Española-Revista de Economía Pública, 177(2), 107-128.

Saad, N. (2012). Tax Non-Compliance Behaviour: Taxpayers View. Procedia-Social and Behavioral Sciences, 65, 344-351.

Schmölders, G. (1951). Finanzpsychologie. FinanzArchiv, 13(1951/1952 H1), 1-36.

Torgler, B. (2005). Tax morale in Latin America. Public Choice, 122(1-2), 133-157.

Torgler, B. (2003). Tax morale: Theory and empirical analysis of tax compliance (Tesis doctoral). University of Basel, Basilea, Suiza.

Torgler, B. (2007). Tax Compliance and Tax Morale: A Theoretical and Empirical Analysis. Cheltenham, Reino Unido: Edward Elgar Publishing Limited.

Torgler B. y Schneider, B. T. (2007). Shadow Economy, Tax Morale, Governance and Institutional Quality: A Panel Analysis. CREMA Working Paper Series 2007-02, Center for Research in Economics, Management and the Arts (CREMA).

\section{Notas}

[1] La EU-15 incluye Austria, Bélgica, Dinamarca, Finlandia, Francia, Alemania, Grecia, Irlanda, Italia, Luxemburgo, Países Bajos, Portugal, España, Suecia y el Reino Unido.

[2] En paréntesis el número de países analizados.

[3] Aunque se excluyan los bancos y se incluyan otras variables institucionales el resultado es el mismo.

[4] Uno de los problemas que deben afrontar las organizaciones internacionales de asistencia sanitaria es que no pueden pedir dinero para inaugurar letrinas, aunque la política de letrina de los años 90 salvó más vidas que los hospitales universitarios a los que los pobres no acuden porque no podrían financiar una enfermedad sofisticada.

La Universidad de Cuenca en Ecuador, conserva los derechos patrimoniales (copyright) de las obras publicadas, y favorece y permite la reutilización de las mismas bajo la licencia Creative Commons AtribuciónNoComercial-CompartirIgual 4.0 Internacional (CC BY-NC-SA 4.0), por lo cual se pueden copiar, usar, difundir, transmitir y exponer públicamente, siempre que: a. Se cite la autoría y fuente original de su publicación (revista, editorial, URL y DOI de la obra). b. No se usen para fines comerciales u onerosos. c. Se mencione la existencia y especificaciones de esta licencia de uso.

CC BY-NC-SA

\section{INFORMACIÓN ADICIONAL}

\section{Código JEL: H26, H21, D15, N16}

Conflicto de intereses: Los autores manifiestan no tener conflictos de intereses. Las opiniones expresadas son exclusivamente de los autores y no se puede considerar en ningún caso como una posición oficial del Banco Solidario. 